\title{
Enhancing Experience Management and Process Learning with Moderated Discourses: the indiGo Approach
}

\author{
Klaus-Dieter Althoff ${ }^{1}$, Ulrike Becker-Kornstaedt ${ }^{1}$, Björn Decker ${ }^{1}$, \\ Andreas Klotz ${ }^{2}$, Edda Leopold ${ }^{2}$, Jörg Rech ${ }^{1}$, Angi Voss ${ }^{2}$
}

\begin{abstract}
The indiGo project aims at improving process knowledge by successive consolidation of feedback, ranging from private annotation, through structured communication in communities of practice, to improved process models and lessons learned. It develops a methodology and integrates previously independent software for process modeling, moderated discourses, experience management and text mining. Both will be evaluated in case studies.
\end{abstract}

\section{INTRODUCTION}

The business process models of organizations operating in innovative, knowledge-intensive or service-oriented markets are one of their major knowledge assets and a competitive advantage. However, these models need to be constantly evaluated and hardened in the business of those organizations and enhanced by further knowledge to make them operable.

The approach of the project indiGo ${ }^{3}$ (Integrative Software Engineering using Discourse-Supporting Groupware) is to support this evaluation and enhancement offering members of an organization to engage in discourses about the process models and their execution (communities of practice) and by presenting process-related lessons learned fitting to the current project context. On the organizational level, finished discourses will be analyzed and summarized to improve process models (process learning) and create new lessons learned (learning from experience).

To achieve these objectives, indiGo will develop an integrated, comprehensive set of methods and a technical infrastructure as a joint effort of two Fraunhofer Institutes: Fraunhofer IESE (Institute for Experimental Software Engineering) in Kaiserslautern and Fraunhofer AIS (Autonomous Intelligent Systems) in Sankt Augustin.

\section{THE FRAMEWORK}

indiGo's key objective is to create and sustain living process models, that is, process models that are accepted by the organizations members, adapted to organizational changes on demand, and continuously enriched with experience from the operating business of the organization.

\subsection{Example}

For example, assume Ms. Legrelle, a team leader in the organization, has to compose an offer for a subcontract from a small start-up. The process model for the acquisition of industrial projects has a subprocess devoted to the contract. It suggests that the payment scheme should not be too finegrained in order to minimize administrative overhead. Ms. Legrelle feels uncomfortable with this guideline. The year before she had had a subcontract with another start-up, Orion, which got bankrupt, so that the last payment was lost for her team although they had completed the work. Ms. Legrelle prefers to design the new offer with a frequent payment schedule, at the cost of more overhead in the administrative unit.

Clearly, Ms. Legrelle would not like to modify the organization's process model (1) for industrial project acquisition on her own - it is not her job and her view may be too subjective. She would probably agree that her experience with the Orion project be recorded as a lesson to be learned, but even so, she would hardly take the trouble to fill in the required form to create an "official" case (2). Rather, she would like to suggest her exception from the guideline to her colleagues, backed up by the example of Orion, and wait for their responses (3). Whatever the conclusion, she would probably add it as a personal note (4) to the guideline in the respective subprocess. A discourse is a deliberative, reasoned communication, it is focused and intended to culminate in group decision making (Erickson 1999). An e-discourse is text-based and conducted (partially) through internet technology. In e-discourses, more persons can participate, the audience may be distributed in space and time, vary in size, composition, background. However, in today's web-based discussion forums, a high tendency to incoherence, drift, and dissolution can be observed. To bridge this tradeoff between promise and reality additional value must be created, and this should be done by exploiting the persistent nature of ediscourses: they may be browsed, replayed, searched, annotated, visualized, analyzed, restructured, and recontextualized.

\footnotetext{
${ }^{1}$ Fraunhofer IESE, Sauerwiesen 6, D-67661 Kaiserslautern, althoff@iese.fraunhofer.de

${ }^{2}$ Fraunhofer AIS, Schloss Birlinghoven, D-53754 Sankt Augustin, angi.voss@fraunhofer.de
} 


\subsection{Knowledge compaction, usage and construction}

indiGo takes into account all four kinds of knowledge occurring in the example and supports them as successive stages in a process of knowledge compaction (aggregation, condensation, summarization, or classification). Figure 1 arranges the four knowledge categories on one layer and embeds it into layers of knowledge usage and knowledge construction.

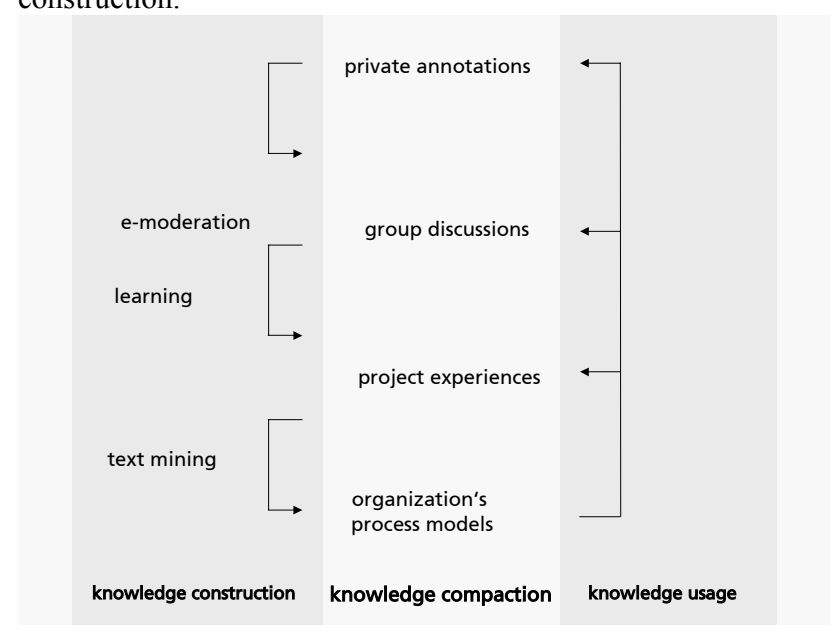

Figure 1. Layers of knowledge compaction, usage and creation for process-centered applications

Knowledge compaction is a process of decontextualization (a) and formalization (b) with the goal of decreasing modification times (c) as well as increasing lifetime (d) and obligingness (e); and of course more obliging knowledge should be more visible (f). As indicators of knowledge compaction (a-f) are correlated, and they exhibit a clear progression from private annotations to group discussions, to stored cases, to an organization's process models. Private annotations are highly contextualized, informal, secret, and non-binding, they have a short lifetime and can be updated often, while process models are highly decontextualized, formal, public, and obliging, they have a long lifetime and are updated infrequently.

The central issue in knowledge usage is how to offer the right knowledge at the right time. As the domain of indiGo is dominated by process models, they should form the backbone for knowledge delivery. While applying (instantiating) a particular process model, members of the organization should find - a mouse click away - supplementary knowledge in associated cases that are dynamically retrieved with regard to the users' current project context. The supplementary knowledge is provided through associated discussions in the users' groups and in their private annotations.

3 indiGo (http://indigo.fhg.de) is funded by the German Ministerium für Bildung und Forschung under grant number 01 AK 915 A
If no relevant knowledge is available, the users have encountered a gap in the knowledge. If they know a solution themselves, they may write a quick private note and attach it to the current part of the process model. Otherwise, they may raise the problem in one of their discussion groups. Other users may be able to help, possibly they had been confronted with a similar problem formerly and had written a private note to remember the solution. Then they may bring this note into the group discussion.

Either way, if a new solution turns up and stands its test, it may be added as a new case to the experience base. The process model would be adapted periodically as substantial feedback is accumulated from the discussions and the new experiences. Again, contributing new bits of knowledge should be a matter of very few mouse clicks.

To extract knowledge from a discussion for the experience base, the indiGo system will be enhanced by text mining tools, and the experience base should offer analytic tools that cluster, categorize, or differentiate the cases as input for improving the process models.

On the one hand, indiGo is more comprehensive than approaches to experience management like (Althoff et al. 2001, Tautz 2000, Bergmann 2001, Minor \& Staab 2002) because it bridges the gap between informal, communicationoriented knowledge and formal, organization-oriented knowledge and provides a socio-technical solution that covers individual knowledge usage as well as social knowledge creation. On the other hand, indiGo is more focused than comprehensive approaches to organizational learning like ENRICH (Mulholland et al. 2000).

\section{THE SOFTWARE PLATFORM}

The indiGo technical platform integrates two independent types of systems for a completely new service. While one system acts as a source for documents, like descriptions of business process models, the other acts as a source for related information, like private annotations, public comments or lessons and examples from an experience base. The business process model repository CoIN-IQ acts as the document source, related information is provided by the groupware Zeno or the experience management system CoIN-EF (Althoff et al. 1999).

Figure 2 shows the components of the indiGo platform as planned for the final version. This paper will focus on the version presented at CeBIT 2002, which comprises an integrator, CoIN-IQ, and Zeno. 


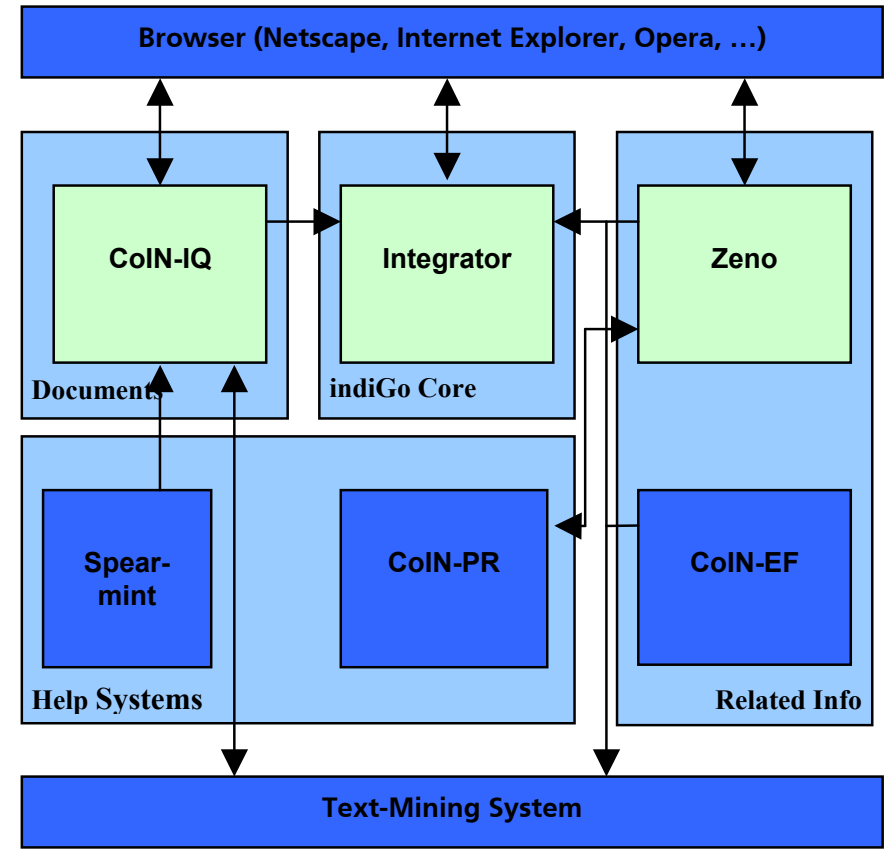

Figure 2. Information flow in the indiGo platform (upper level presented at CeBIT 2002)

The integrator acts as a middleware between the document and information source. On the left hand side CoIN-IQ, as the document source, hosts the business process models that can be supported by the information from the second system. Zeno, as the information source on the right side, manages annotations and discussions about the business process models from CoIN-IQ.

To enhance the functionality of indiGo we connected Zeno with CoIN-PR (CoIN Project Registry), a project repository that stores all information about the projects and associated users. Information about the projects include, for example, the project type (e.g., research \& development, transfer, or consulting), status, funding, project staff, project manager, or the list of participating partners.
CoIN-PR delivers information about a specific user's current projects, which is used to index contributions in Zeno with a project context and to construct queries for CoIN-EF. Beside commenting the business process models, the user will have the opportunity to recall context-specific lessons learned from CoIN-EF. To support and enhance the various roles in indiGo text-mining tools will be applied to analyze the discussions in order to detect new, previously unknown or hidden information for moderators and other roles, especially with the goal to extend or improve the lessons learned and the process models.

Based on standard internet technology indiGo is a truly distributed system. While Zeno is hosted on a web server at Fraunhofer AiS in Sankt Augustin, Germany, the CoIN system family is located at and maintained by Fraunhofer IESE in Kaiserslautern, Germany.

\subsection{The integrator}

The integrator is the glue between a document server like CoIN-IQ and a server for related information like Zeno. It provides an integrated view upon a document and related information (see figure 3). Based on Perl the integrator is a CGI script that offers three fundamental functions that are called either by CoIN-IQ or Zeno:

- Discuss: This function creates a split view upon a document and related information. In the current indiGo context this is a view on the specific business process model from CoIN-IQ in the upper part and beneath the appropriate discussion from Zeno.

- Annotate: Analogous to the previous function, the integrator creates a split view upon a business process model and a personal annotation for the current user.

- Destroy: To work with only one system this function collapses the split view of indiGo to a single frame. This is particularly helpful if the user wants to turn off the discussions from Zeno or if he switches into another discourse in Zeno that is not related to business processes. 


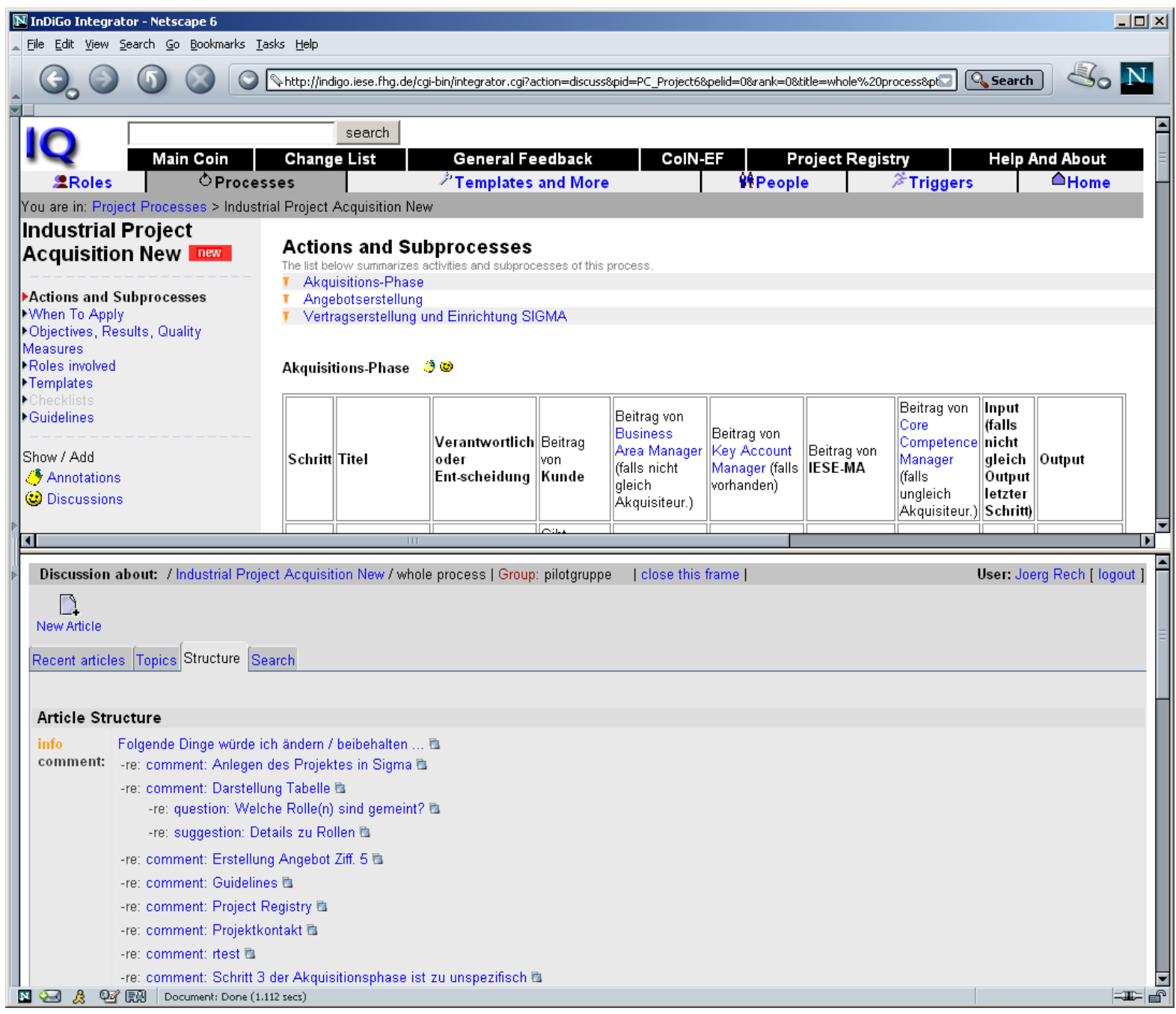

Figure 3. Split View with CoIN-IQ at the top and a related discussion in Zeno beneath

\subsection{CoIN-IQ}

CoIN-IQ is IESE's business process model repository (Decker and Jedlitschka, 2001). The topics currently covered range from core processes (e.g., project set-up and execution) to support processes (e.g., using the IESE information research service) to research focused processes (e.g., performing Ph.D. work at IESE).

\subsubsection{Baseline}

The objectives of CoIN-IQ can be positioned according to four criteria: (1) The purpose of process models, (2) the origin and (3) usage of the process models, and (4) the modeling techniques. In summary, CoIN-IQ uses structured text describing empirical and theoretical process models to be executed by human agents. This is detailed in the following.
For the general purpose of process models, Curtis, Kellner, and Over (1992) identify five different categories: Facilitate human understanding and communication, support process improvement, support process management, automate process guidance, and automate execution. According to this classification scheme, CoIN-IQ fits into the first category of facilitating human understanding and communication: The processes are executed by human agents (i.e., IESE members), based on the process description. Supporting and enforcing process execution beyond this human-based approach (e.g., by workflow modeling and enactment as in (Maurer and Holz 1999)) was regarded as non-suitable for the purposes of IESE due to the creative nature of its business processes. Furthermore, processes according to the process models are executed rather infrequently ( $<10$ times per month), therefore (a) automation of the processes was not supposed to leverage a high cost/benefit and (b) tracking of process status can be done by asking the responsible process executor. In addition, the experience made with the Electronic Process Guide (EPG) (Becker-Kornstaedt \& al. 1999) showed that web-based 
process descriptions are a feasible way of distributing process knowledge within creative environments such as software business. In particular, changes to web-based process models can be communicated much quicker than paper-based process models, thus enabling quick integration of experience.

The origin of process models can be empirical (i.e., based on actual processes (Bandinelli, Fugetta et. al 1995)) and theoretical (i.e., reflecting a planned process execution). Process models in CoIN-IQ have both origins: Some of the process models reflect well-established processes (like, e.g., the administrative project set-up), others represent new procedures (e.g., the reflection of recent changes in the organizational structure of IESE).

The usage of process models can be descriptive (i.e., a description of a process) or prescriptive (i.e., intended to be used as an instruction for process execution). The process models within CoIN-IQ are prescriptive with different degrees of obligation. In general, administrative procedures (e.g., project accounting) have to be followed without exception; best-practice process models like project management procedures are to be seen as recommendations.

The process modeling technique of CoIN-IQ is structured text, which is due to several reasons: Zero effort training, straightforward modeling, and perpetuation in industrial strength applications. Zero effort has to be spent on training, since any IESE member can read structured text without previous training. Furthermore, straightforward modeling means that any IESE members can model processes using structured text, if supported by guidelines and the CoIN team. This aspect is additionally fortified by the experience in scientific publishing of most IESE members.

\subsubsection{Concepts}

To achieve these objectives, the following information is captured within CoIN-IQ:

- Process descriptions describe the activities captured within CoIN (e.g., project management). Complex processes are structured into a hierarchy of super- and sub-processes.

- Role descriptions describe the roles that are involved in the execution of processes.

- Agent descriptions are used within role descriptions to name roles that are performed by a specific IESE member.

- Product representations represent a document to be used during process execution.

- Overviews structure the other objects within CoIN-IQ to facilitate browsing.
The discussions in indiGo are related to process descriptions, which consist of "Actions and Subprocesses", "When to apply?", "Objectives, Results, and Quality Measures", "Roles involved", "Templates", "Checklists", and "Guidelines" (see Figure 4).

"Actions and Subprocesses" describe the steps of the process execution. In CoIN-IQ, a distinction is made between actions and sub-processes. Actions are atomic steps that are not refined any further. Sub-processes are described in a separate process description according to this structure. The superprocess contains a link to the sub-process, followed by a short explanation of the sub-process content.

"When to Apply" gives a short overview of a process' context, thus helping the user to determine if the current process description is the desired one. To facilitate this overview even more, it is again structured into three sub-sections: Scope, Trigger and Viewpoint. "Scope" contains one or two sentences about the thematic range of a process and thus, the content of a process description. "Trigger" as the second sub-section describes the condition that starts the execution of a process. These triggering conditions can be events released from outside IESE (e.g., a customer telephone call), dependencies with other process executions (e.g., start or finish of a process) or dependencies from product states (e.g., a deliverable is about to be finished). "Viewpoint" contains the role from whose view the process is described.

"Objectives, Results and Quality Measures" is information intended to guide the execution of a process. The difference between the three sub-sections is the increasing degree of quantification of quality information. "Objectives" are general objectives of the process. "Results" are tangible outcomes of the process (e.g., meeting minutes). "Quality Measures" describe properties of such results (e.g., the number of pages of the meeting minutes should range between 10 and 20) or the process itself (e.g., the effort spent on preparing a meeting should not exceed one person day).

"Roles involved" provides an overview of the roles involved in the process and links the Role Descriptions. An experienced user can quickly find the Role Descriptions that are distributed within the "Actions and Subprocesses" and "Guidelines" Section.

"Templates" lists the products referenced by the process description. This overview is intended to support IESE members, who are accustomed to the process and just need quick access to artifacts.

"Checklists" is also intended for the experienced user. It summarizes important steps and results of the Process Description. 
"Guidelines" give hints for performing a process, like "do's and don'ts" or frequently asked questions about a process. Furthermore, frequently used variances of a process are modeled as guidelines. This reduces the number of similar process descriptions and lowers the effort to maintain the process description. Each guideline has a "speaking headline" in the form of a question or statement, followed by explanatory text.

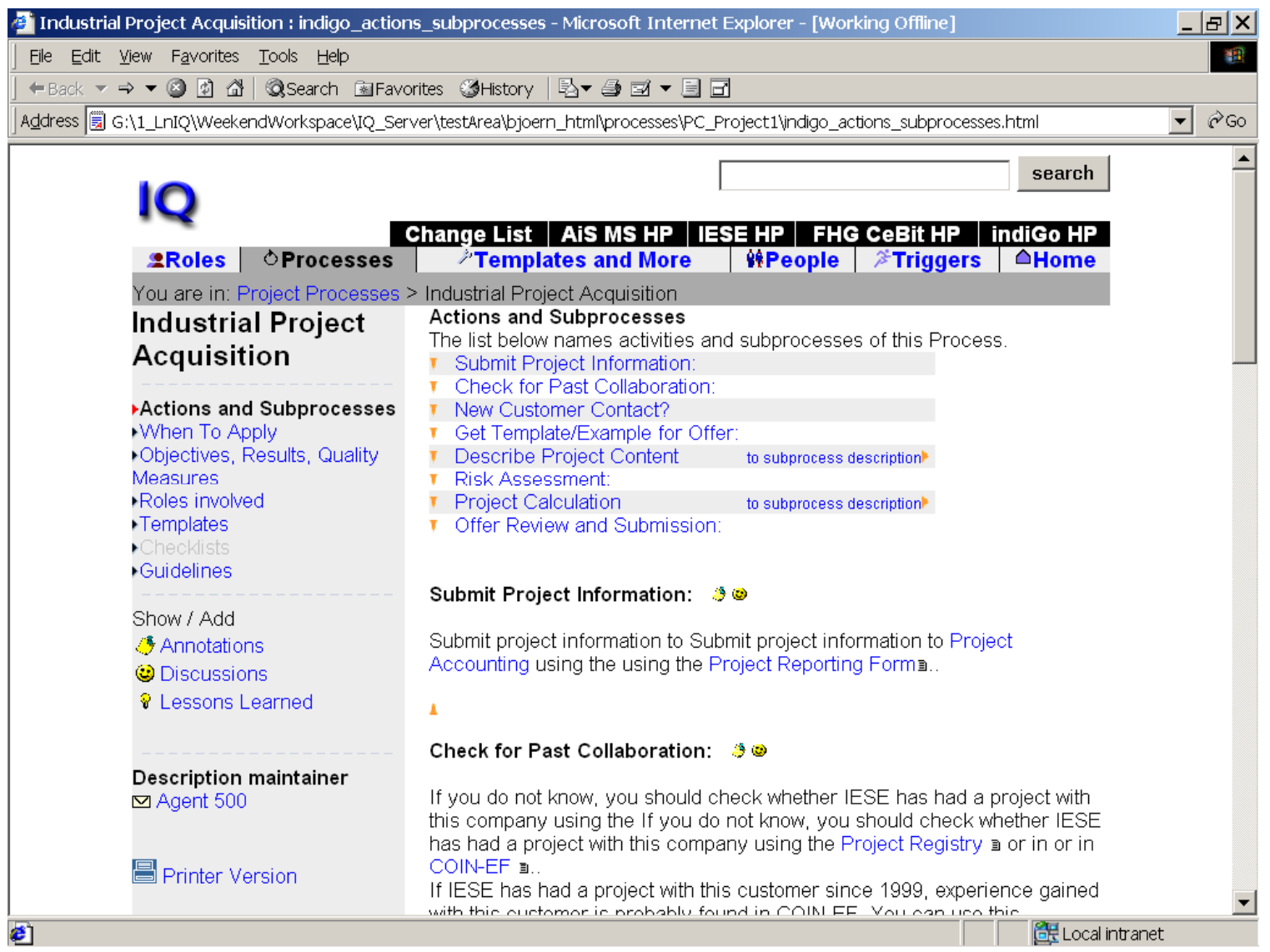

Figure 4. Screenshot of a process description. (Figure shows anonymized demonstrator)

\subsubsection{Integration}

In the indiGo platform, CoIN-IQ's start page is automatically generated by Zeno from articles in a special section for announcements. Other modifications of CoIN-IQ for indiGo concern the insertion of buttons for private annotations, group discussions, and lessons learned. The buttons are displayed or hidden at the user's discretion. Buttons are inserted for entire processes and for all process elements. Internally, each process and element is identified by a unique number for the indiGo integrator and the other components; this number will not change even if the process model is reorganized.

\subsection{Zeno}

Zeno is an e-participation platform (www.e-partizipation.org) (Voss 2002) with a spectrum of functions for moderated discourses on the web.

\subsubsection{Baseline}

Zeno comprises and extends (1) simple threaded discussions, (2) document-centered discourses, and (3) information structuring during group decision making.

Most electronic discussion forums, like the ones mentioned above but also newsgroups, support simple threaded discussions (1). Some tools, e.g. http://icommons.harvard.edu/, recognize URLs or even HTML tags in the contributions or allow to attach documents.

$\mathrm{D}^{3} \mathrm{E}$ belongs to category (2). It can process any hierarchical HTML file into a frames-based environment with automatic hyperlinking for navigating around sections, checking citations and footnotes, and tight integration with a discussion space for critiquing documents. Moderators may influence the look and feel of a discussion space, they may edit, hide, or delete contributions. $\mathrm{D}^{3} \mathrm{E}$ is available as open source (http://d3e.sourceforge.net/) (Sumner \& Buckingham Shum 1998). The e-learning platform Hyperwave eLearning SUITE 
supports annotations and discussions of course units. Moreover, it offers a set of labels to characterize contributions as notes, questions, responses, acceptance, and rejection (www.hyperwave.com).

Predefined labels for qualifying contributions are more familiar in tools for group decision making (3), especially for brainstorming (www.facilitate.com). Softbicycle's QuestMap (www.softbicycle.com) distinguishes questions, ideas, pros, cons, decisions, notes, and references, a variant of the famous IBIS grammar (Kunz \& Rittel 1970) which was first implemented in gIBIS (Conklin \& Begemann 1988). Tools in this category usually allow to restructure the contributions, that is, they support maps rather than threads, deliberative argumentation rather than spontaneous reaction.

The first version of Zeno, which also supported a variant of IBIS (Gordon \& Karakapilidis 1999), was presented at CeBIT 1996 and continuously improved up to version 1.9 in 1999. Since then a completely new system has been realized that addresses a broader spectrum of discourses in the knowledge society: Participatory problem solving, consensus building (Voss, Röder \& Wacker, 2002), mediated conflict resolution (Märker, O., Hagedorn, H., Trénel, M. \& Gordon, 2002), teaching, and consulting. The new Zeno focuses on ediscourses and supports e-moderators in turning discussions into discourses, elaborating the argumentation, and carving out rationales.

A discourse is a deliberative, reasoned communication; it is focused and intended to culminate in decision making
(Erickson 1999). (Turoff et al. 1999) argued that building a discourse grammar, which allows individuals to classify their contributions into meaningful categories, is a collaborative effort and its dynamic evolution is an integral part of the discussion process. A discourse grammar (or ontology) defines labels for contributions, labels for references (directed links) between contributions, and may constrain links with respect to their sources and targets. Supporting communities in evolving their own discourse grammars has been a key issue in the design of Zeno.

\subsubsection{Concepts}

As a consequence, Zeno distinguishes three kinds of objects: Sections to tailor the settings for an e-discourse, articles as units of a communication (contributions), and links as directed relations between articles or even sections (see Figure 5). 


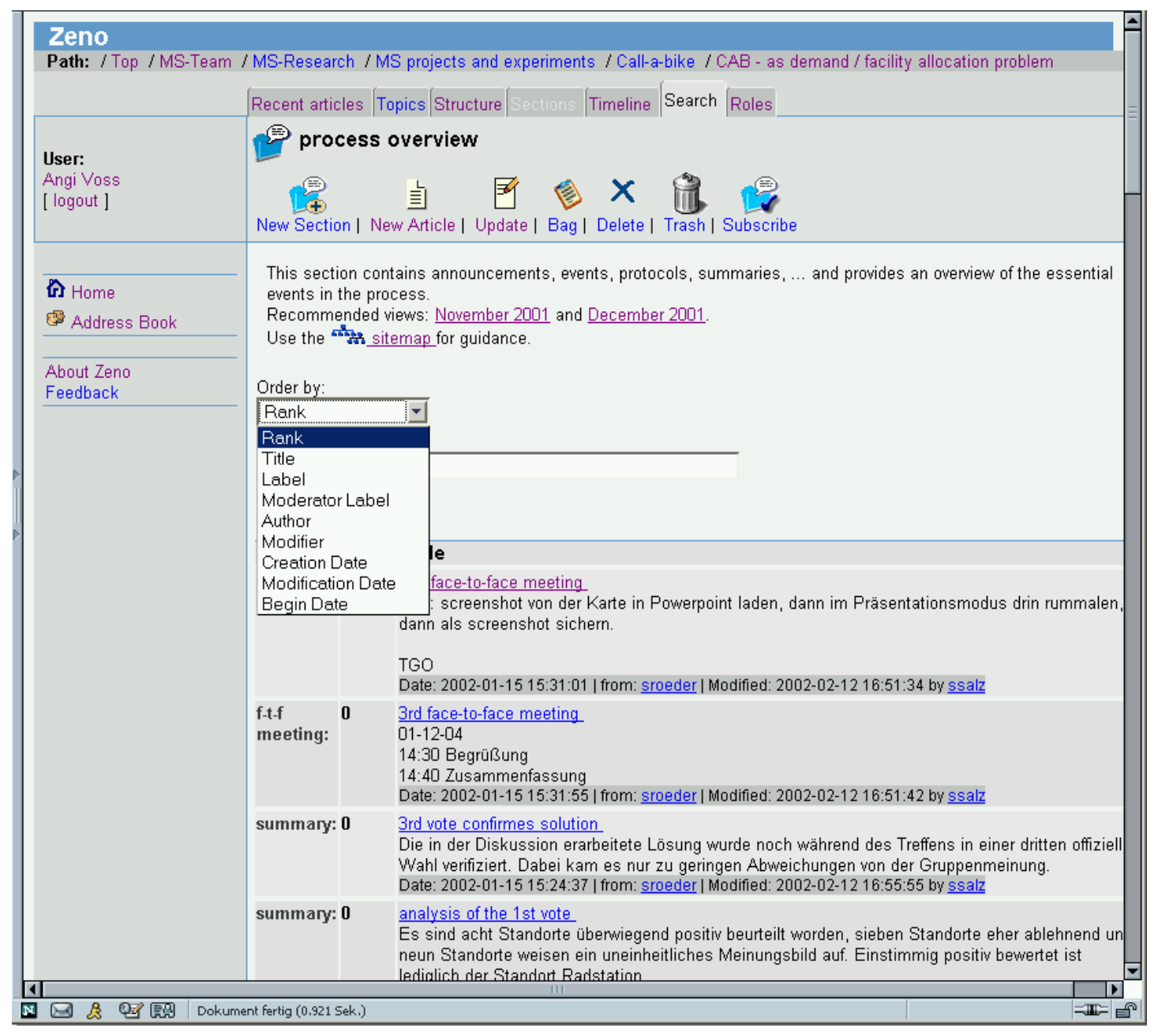

Figure 5. The search view in the overview section of a spatial decision making discourse in Zeno

Moderators specify the readers, authors, and co-editors of the section, its discourse grammar, a style sheet to control the presentation, and plugged-in functionality (for mapping, awareness, polling, etc).

An article has a title, usually a note (plain text or html), and possibly document attachments. From its author it may get a label to indicate its pragmatic (or ontological) role in the discourse (e.g. issue, option, criterion, argument, decision, summary, question, comment), and it may receive an additional qualifier from the moderator (e.g. green, yellow, red cards). Articles may be selected (and deselected) as topics and may be ranked to influence their ordering. An article may have temporal references (to be displayed on a timeline), keywords (to be searched together with the title and note), and attributes related to its visibility and accessibility.

Links between articles or sections may be labeled to express relations, such as refers-to, responds-to, justifies, questions, generalizes, suggests, pro, contra) so that complex networks (or hyperthreads) can be built. Links between Zeno articles and sections are visible at both end points and can be traversed in both directions. They are automatically maintained by
Zeno, so moderators may edit, copy, and move groups of articles with their links.

Zeno links may also point to external web resources; they are used for document references in indiGo and for spatial references (to be displayed on a map) in KogiPlan (www.kogiplan.de).

Users are received on a personal home page. Here they can bookmark and subscribe sections in order to be notified of their latest contributions. Each section offers different views: The latest articles, the topics, the complete article structure, a sorted list of articles as a result of a full-text search, the hierarchy of subsections, or the timeline. Authors may create or respond to articles in a section, and moderators may edit, move and copy articles, change links and assign labels, and manipulate sections. Users and groups are administered through an address book.

Zeno can be accessed from any regular web browser without any local installations. The Zeno server is implemented on top of open source products: tomcat as web server and servlet runner, velocity for templates in the user interface, Java for the 
kernel, and MySQL for the data base. Zeno itself is available as open source (http://zeno.berlios.de/).

\subsubsection{Integration}

In Zeno, document-centered discourses, or more specifically, discourses about process models, are made possible through the indiGo integrator and some indigo-specific adaptations of Zeno.

The structure and ordering of process models and their elements is reflected in the hierarchies of sections and their ranking. The mapping between these structures is accomplished through Zeno links, the names of which encode identifiers for the process model and element.

Moderators first create entries for users and groups in the address book. Next, to generate a section for discussing a process, the moderators click on the "discussion" button of the process or any of its elements and then select a group as readers and writers for the discussion. Subsections for discussing process elements are created on demand, when users click on the associated processes and selects the discussion group. The subsections inherit the discourse grammar of their super-section and are restricted to the selected group as authors.

When a user clicks on an "annotation" button for the first time, a personal section is created. This section and its subsections can only be accessed by this user with all rights of a moderator. Subsections for processes and their elements are again created on demand, when the user clicks on the corresponding "annotation" buttons.

The start page of the indiGo system is automatically generated. The upper part displays announcements. These are articles in a section called "StartPage", can be edited by all indiGo moderators. Beneath the announcements, the start page lists all new articles in the user's discussion groups. This service replaces the subscription and notification mechanism that is otherwise available on the users' personal home page in Zeno. For the introduction and operational phases different discourse grammars will be available. "info", "question", "comment", "suggestion", "example" are the article labels during introduction, "observation", "problem", "suggestion", "solution", "example" and "summary" are the article labels during operation. Link labels are in both phases "re", "pro", "con", "see also". Qualifier will include "closed" to indicate threads with a conclusion, and "invalid" to indicate threads that may have become invalid due to modifications of the process model. To come back to the introductory example, Ms Legrelle could have attached a "problem" to the guideline on payment schedules, "re"sponded with a "suggestion" concerning small start-ups, and supported it with a "pro" "example" from the Orion project.

\section{CONCLUSIONS AND OUTLOOK}

indiGo aims at supporting all kinds of knowledge that have been identified as being import for process learning, namely process models (with their associated templates), experiences from instantiating process models in concrete projects, discussions about processes in closed or open groups, and private annotations of process models. Thus, with indiGo, any concerned organization member can make private annotations for a newly introduced, or changed, business process model. Staff can decide which of the issues that attracted their attention should be discussed within a selected group of people.

This paper focused on the technical infrastructure of indiGo, as presented at CeBIT 2002. It enables the organization of various process-related annotations and moderated discussion groups based on a customizable discourse grammar.

How an organization can accomplish process learning using the indiGo platform is the core of the indiGo methodology. In (Althoff et al. 2002) the methodology is described in more detail. It is itself phrased as a set of process models. The selfdescription of the indiGo methodology through indiGo process models offers the opportunity to 'bootstrap' indiGo, that is, to apply indiGo to itself. First, it allows having a test run of both the methodology and the technical infrastructure during the introduction of indiGo. Furthermore, since the persons involved in the indiGo introduction directly perform and experience this approach, it will be their prime interest to resolve occurring difficulties. Therefore, the members of the organization can rely on a tested infrastructure and a consolidated team to support them in the roll-out phase.

In April 2002, the indiGo case study has been started, carried out at Fraunhofer IESE in Kaiserslautern, Germany. New project and research processes will be introduced for the whole institute. We expect very valuable feedback for all the described indiGo methods and technologies.

In parallel, work on the software platform is progressing with specified but not yet implemented features. For instance, if a process model is modified or reorganized, the corresponding annotations and discussions should automatically be marked for re-validation or be reorganized accordingly. Next, the components indicated in Figure 2 will be integrated, starting with CoIN-EF.

indiGo's e-moderation method guarantees that discussions are carried out in a structured and goal-oriented manner. This helps to identify valuable experiences, which then are represented as semi-formal cases, and stored in the experience base. Using case-based reasoning, these experiences are then available for both process improvement/change and process execution.

As soon as discussions will become available from the case study, text mining experiments can begin (Kindermann et al. 2002, Leopold and Kindermann 2002). For that purpose, the discussions in Zeno will be exported in GXL, an XML dialect for graph structures. Private annotations remain private and will not be subject to text mining.

Beyond the current project we consider the possibility to extend the indiGo approach to applications where process models do not play such a central ("backbone") role. Although a platform for organizational learning should eventually cover all knowledge categories treated in indiGo, the first steps to organizational learning need not necessarily involve process models. Maybe, an organization would first like to invest into an experience base or into a communication platform, and add 
process models only later. The challenging research question here is, to which degree indiGo's methods and technologies can still be applied or easily tailored to such an organization's needs.

\section{REFERENCES}

Althoff, K.-D., Becker-Kornstaedt, U., Decker, B., Klotz, A., Leopold, E., Rech, J., Voss, A. (to appear 2002): "The indigo project: enhancement of experience management and process learning with moderated discourses". In Data mining in marketing and medicine (Ed. P. Perner), Springer Verlag, LNCS.

Althoff, K.-D.; Birk, A.; Hartkopf, S.; Müller, W.; Nick, M.; Surmann, D. Tautz, C.: Managing Software Engineering Experience for Comprehensive Reuse; Proceedings of the Eleventh Conference on Software Engineering and Knowledge Engineering, Kaiserslautern, Germany, June 1999; Knowledge Systems Institute; Skokie, Illinois, USA; 1999.

Althoff, K.-D., Decker, B., Hartkopf, S., Jedlitschka, A., Nick, M. \& Rech, J. (2001). Experience Management: The Fraunhofer IESE Experience Factory. In P. Perner (ed.), Proc. Industrial Conference Data Mining, Leipzig, 24.-25. Juli 2001, Institut für Bildverarbeitung und angewandte Informatik

Althoff, K.-D., Feldmann, R. \& Müller, W. (eds.) (2001). Advances in Learning Software Organizations. Springer Verlag, LNCS 2176, September 2001.

Bandinelli, S., Fuggetta, A., Lavazza, L., Loi, M. \& Picco G.P. (1995). Modeling and improving an industrial software process, Transactions on Software Engineering, 440-454.

Basili, V.R.; Caldiera, G.; Rombach, D.: Experience Factory; In Marciniak, J.J. ed., Encyclopedia of Software Engineering, vol 1, 469-476; John Wiley \& Sons; 1994.

Ulrike Becker-Kornstaedt, Dirk Hamann, Ralf Kempkens, Peter Rösch, Martin Verlage, Richard Webby, and Jörg Zettel. Support for the Process Engineer: The Spearmint Approach to Software Process Definition and Process Guidance. In Proceedings of the 11th Conference on Advanced Information Systems Engineering (CAISE '99), Heidelberg, Germany, June 1999. Lecture Notes on Computer Science, Springer-Verlag.

Bergmann, R. (2001). Experience management - foundations, development methodology, and internet-based applications. Postdoctoral thesis, Department of Computer Science, University of Kaiserslautern.

Conklin, J. and M. Begeman (1988) "gIBIS: A Hypertext Tool for Exploratory Policy Discussion", Transactions of Office Information Systems, 6(4): 303-331.

Curtis, B., Kellner, M. I., and Over, J. (1992). Process modeling. Communications of the ACM, 35(9):75-90, Sept. '92

Decker, B. \& Jedlitschka, A. (2001). The Integrated Corporate Information Network iCoIN: A Comprehensive Web-Based Experience Factory. In Althoff, Feldmann \& Müller (2001), 192206.

Erickson, T. (1999). Persistent Conversation. An introduction. Journal of Computer-Mediated Communication, 4.

Gordon, Thomas F., und Nikos Karacapilidis. 1997. "The Zeno Argumentation Framework." Pp. 10-18 in Proceedings of the Sixth International Conference on Artificial Intelligence and Law.

Marc I. Kellner, Ulrike Becker-Kornstaedt, William E. Riddle, Jennifer Tomal, and Martin Verlage (1998). Process Guides: Effective Guidance for Process Participants. In Proceedings of the Fifth International Conference on the Software Process, pages 11-25, Chicago, IL, USA, June 1998. ISPA Press.
Kindermann, Jörg \& Diederich, Joachim \& Leopold, Edda \& Paaß, Gerhard (2002): Identifying the Author of a Text with Support Vector Machines; accepted at Applied Intelligence.

Kunz, Werner und Horst W.J. Rittel (1970): "Issues as elements of information systems." Center for Planning and Development Research, Institute of Urban and Regional Development Research. Working Paper 131, University of California, Berkeley.

Leopold, Edda \& Kindermann, Jörg (2002): Text Categorization with Support Vector Machines. How to Represent Texts in Input Space?; in: Machine Learning 46, 423 - 444.

Märker, O., Hagedorn, H., Trénel, M. and Gordon, T. F. 2002 'Internet-based Citizen Participation in the City of Esslingen. Relevance - Moderation - Software', in M. Schrenk (ed) CORP 2002 - "Who plans Europe's future?" Wien: Selbstverlag des Instituts für EDV-gestützte Methoden in Architektur und Raumplanung der Technischen Universität Wien.

Maurer, F. \& Holz, H. (1999). Process-Oriented Knowledge Management For Learning Software Organizations, Proceedings of 12th Knowledge Acquisition For Knowledge-Based Systems Workshop 1999 (KAW99); Banff, Canada.

Minor, M. \& Staab, S. (eds.) (2002). $1^{\text {st }}$ German Workshop on Experience management - Sharing Experiences about the Sharing of Experience, Berlin, March 7-8, 2002, Lecture Notes in Informatics, Gesellschaft für Informatik (Bonn).

Mulholland, P., Domingue, J., Zdrahal, Z., and Hatala, M. (2000). Supporting organisational Learning: An Overview of the ENRICH Approach. Journal of Information Services and Use, 20 (1) 9-23.

Sumner, T. \& Buckingham Shum, S. (1998) "From Documents to Discourse: Shifting Conceptions of Scholarly Publishing“, Proc. CHI 98: Human Factors in Computing Systems, 18-23 April, 1998, Los Angeles, CA. ACM Press: New York

Tautz, C. (2000). Customizing Software Engineering Experience Management Systems to Organizational Needs. Doctoral Dissertation, Department of Computer Science, University of Kaiserslautern, Germany.

Turoff, M., Hiltz, S. R., Bieber, M., Fjermestadt, J. and Ajaz, R. (1999): "Collaborative discourse structures in computer-mediated group communications". Journal of Computer-Mediated Communication, 4.

Voss, A. (to appear (2002)): „Zeno - Software für Online-Diskurse in der Mediation". In Online-Mediation. Theorie und Praxis computerunterstützter Konfliktmittlung(Eds, Märker, O. and Trenél, M.) Sigma, Berlin.

Voss, A., Roeder, S. and Wacker, U. (to appear (2002)): „IT-support for mediation in decision making - A role playing experiment”. In Online-Mediation. Theorie und Praxis computer-unterstützter Konfliktmittlung.(Eds, Märker, O. and Trenél, M.) Sigma, Berlin. 